\title{
Pulsar timing irregularities and the imprint of magnetic field evolution
}

\author{
J. A. Pons ${ }^{1}$, D. Viganò ${ }^{1}$, and U. Geppert ${ }^{2,3}$ \\ 1 Departament de Física Aplicada, Universitat d'Alacant, Ap. Correus 99, 03080 Alacant, Spain \\ e-mail: Jose.Pons@ua.es \\ 2 German Aerospace Center, Institute for Space Systems, , Robert-Hooke-Str. 7, 28359 Bremen, Germany \\ 3 Kepler Institute of Astronomy, University of Zielona Gora, Lubuska 2, 65-265 Zielona Gora, Poland
}

Received 24 July 2012 / Accepted 4 September 2012

ABSTRACT

Context. The rotational evolution of isolated neutron stars is dominated by the magnetic field anchored to the solid crust of the star. Assuming that the core field evolves on much longer timescales, the crustal field evolves mainly though Ohmic dissipation and the Hall drift, and it may be subject to relatively rapid changes with remarkable effects on the observed timing properties.

Aims. We investigate whether changes of the magnetic field structure and strength during the star evolution may have observable consequences in the braking index $n$. This is the most sensitive quantity to reflect small variations of the timing properties that are caused by magnetic field rearrangements.

Methods. We performed axisymmetric, long-term simulations of the magneto-thermal evolution of neutron stars with state-of-the-art microphysical inputs to calculate the evolution of the braking index. Relatively rapid magnetic field modifications can be expected only in the crust of neutron stars, where we focus our study.

Results. We find that the effect of the magnetic field evolution on the braking index can be divided into three qualitatively different stages depending on the age and the internal temperature: a first stage that may be different for standard pulsars (with $n \sim 3$ ) or low field neutron stars that accreted fallback matter during the supernova explosion (systematically $n<3$ ); in a second stage, the evolution is governed by almost pure Ohmic field decay, and a braking index $n>3$ is expected; in the third stage, at late times, when the interior temperature has dropped to very low values, Hall oscillatory modes in the neutron star crust result in braking indices of a high absolute value and both positive and negative signs.

Conclusions. Current magneto-thermal evolution models predict a large contribution to the timing noise and, in particular, to the braking index, from temporal variations of the magnetic field. Models with strong $\left(\gtrsim 10^{14} \mathrm{G}\right)$ multipolar or toroidal components, even with a weak $\left(\sim 10^{12} \mathrm{G}\right)$ dipolar field are consistent with the observed trend of the timing properties.

Key words. pulsars: general - stars: neutron - stars: magnetic field - stars: evolution

\section{Introduction}

The spin-down of an isolated neutron star (NS) on secular timescales is mainly caused by rotational energy losses due to electromagnetic radiation, pulsar winds, or gravitational radiation. A measurable quantity closely related to the rotational evolution of pulsars is the braking index $n$, defined by assuming that the star spins down according to a power law $\dot{\Omega}=-K \Omega^{n}$, where $\Omega$ is the NS angular velocity. For each of these dominant rotational energy loss mechanisms, the proportionality constant $K$ hides different dependences on the star radius, moment of inertia, magnetic field strength, and angle between rotation and magnetic axis. If all these quantities are constant in time, the magneto-dipole spindown mechanism predicts a braking index $n=3$, but variations in time of any of these quantities may cause departures from this canonical value. Unfortunately, the accurate determination of the second derivative of the frequency, needed to estimate the braking index, is not always possible because it is affected by glitches and other short-term timing irregularities.

At present, eight pulsars have sufficiently steady rotations that stable values of their braking index are generally accepted (Lyne et al. 1993, 1996; Middleditch et al. 2006; Livingstone et al. 2007, 2011; Weltevrede et al. 2011; Espinoza et al. 2011), among which the most recent case is PSR J1734-3333, which has $n=0.9 \pm 0.2$ significantly below 3 (Espinoza et al. 2011). All these cases show the same trend: they are all young pulsars
(Vela is the oldest among these pulsars with $t \approx 12 \mathrm{kyr}$ ) and they all have $n<3$. We note that gravitational wave emission predicts $n=5$, but it is only efficient during the first minutes or hours of a NS life, when rotation is sufficiently fast and the mass quadrupole moment large enough (see e.g., Haskell et al. (2006) and references therein). It certainly does not contribute significantly to the timing noise of pulsars older than $100 \mathrm{yr}$.

Selecting a sample of 127 pulsars from the ATNF Pulsar Catalogue (Manchester et al. 2005), for which the quoted errors in the second derivative of the spin frequency $(\ddot{v})$ are less than ten per cent, Urama et al. (2006) found a strong correlation of $\ddot{v}$ with $\dot{v}$, independent of the sign of $\ddot{v}$. They suggested that this trend can be accounted for by small stochastic deviations in the spin-down torque that are directly proportional (in magnitude) to the spin-down torque itself. Another point discussed in the literature is that some of the old pulsars $\left(>10^{6} \mathrm{yr}\right)$ have braking indices with absolute values exceeding $|n|=10^{4}$. The occurrence of very high braking indices of both signs has been considered in the context of internal frictional instabilities occurring between the crust and the superfluid, almost independently of the evolution of the neutron star magnetic field (Shibazaki \& Mochizuki 1995). However, this applies only for old neutron stars $\left(\tau \gtrsim 2 \times 10^{7} \mathrm{yr}\right)$ and appears as extremely short term events oscillating about the canonical value $n=3$. Barsukov \& Tsygan (2010) proposed another explanation for the observed 
distribution with very high positive and negative braking indices by studying the effect of nondipolar magnetic field components and neutron star precession on magnetospheric electric current losses. These large $n$ should be observable over relatively long periods of $10^{3}-10^{4} \mathrm{yr}$. Another possibility that can explain the observed variability of braking indices is the time-evolution of conductivity in the magnetosphere (Li et al. 2012b), which also has implications for the spin-down of intermittent pulsars and subpulse drift phenomena (Lyne 2009).

In this paper we focus on the imprint that the time-evolution of the internal magnetic field has on the timing noise. Our goal is to estimate the contribution of this variability (expected in realistic evolutionary models) to the braking index at different epochs. In particular, we discuss the possible reemergence of a magnetic field initially submerged by hypercritical accretion during the supernova explosion, and the evolution of the crustal magnetic field under the combined action of Ohmic dissipation and Hall drift during the first few million years of a NS life.

\section{Basic equations}

The spin-down behavior of a rotating neutron star is governed by the energy balance equation relating the loss of rotational energy, $E_{\mathrm{rot}}=I \Omega^{2} / 2$, where $I$ is the moment of inertia of the neutron star and $\Omega$ the angular velocity, to the energy loss rate $\dot{E}$, given by magneto-dipole radiation, wind, gravitational radiation, or others:

$\dot{E}_{\mathrm{rot}}=I \Omega \dot{\Omega} \approx \dot{E}$.

The standard way to define the braking index is

$n=\frac{\ddot{\Omega} \Omega}{\dot{\Omega}^{2}}=\frac{\ddot{v} v}{\dot{v}^{2}}=2-\frac{\ddot{P} P}{\dot{P}^{2}}$,

where $v$ is the spin frequency, and we denote by $P=1 / v$ the period. Under the usual assumption of a power-law rotational evolution

$\dot{\Omega}=-K \Omega^{n}$,

the power-law index coincides with the braking index.

For the particular case of a rotating dipole in vacuum, the well-known Larmor formula returns

$\dot{E}=\frac{B_{0}^{2} R^{6} \Omega^{4}}{3 c^{3}} \sin \alpha^{2}$,

where $B_{0}$ is the dipolar component of the surface magnetic field at the pole, $R$ denotes the neutron star radius, $\alpha$ is the angle between the rotational and the magnetic axis, and $c$ is the speed of light. We neglect the contribution to the spindown of higher order multipoles, since they have significantly shorter "lever arms" than the dipolar mode.

Combining Eqs. (1) and (4), one obtains

$\dot{\Omega}=-K \Omega^{3}$,

where $K=f_{\alpha} B_{0}^{2} R^{6} / 3 I c^{3}$. For simplicity, but without loss of generality, we have omitted the geometric factor that carries the dependence on the inclination angle, and we assume hereafter that it is a correction $f_{\alpha}$ factorized in $K$, which carries information about the particular physical process that governs energy losses. The functional dependence of $K$ for all other magnetic processes is the same $\propto B_{0}^{2} \Omega^{4} R^{6}$. Differences in the radiation mechanism are included in the factor $f_{\alpha}$. While magnetospheric current losses scale as $\cos ^{2} \alpha$ (Beskin \& Nokhrina 2007), magneto-dipole radiation losses scale as $\sin ^{2} \alpha$ for vacuum or $\frac{3}{2}\left(1+\sin ^{2} \alpha\right)$ for force-free magnetospheres (Spitkovsky 2006). The most recent resistive solutions for pulsar magnetospheres (Li et al. 2012b) fit the spin-down luminosity with a prefactor of the order of unity that also depends on the maximum potential drop along field lines in the corotating frame.

Equation (5) can also be cast in the usual form

$P \dot{P}=\mathcal{K} B_{0}^{2}(t)$

where for a standard neutron $\operatorname{star}\left(R=10^{6} \mathrm{~cm}, I=10^{45} \mathrm{~g} \mathrm{~cm}^{2}\right)$ the constant $\mathcal{K} \approx 10^{-39} \mathrm{~cm} \mathrm{~s}^{3} \mathrm{~g}^{-1}$. Here we have explicitly written $B_{0}(t)$ to show that the magnetic field is the only quantity that we allow to vary with time.

Now we consider what happens when a secular variation of the magnetic field is allowed. Deriving $\ddot{P}$ from Eq. (6), we have

$\ddot{P}=\mathcal{K} B P^{-2}\left(2 \dot{B}_{0} P-B_{0} \dot{P}\right)$,

and the braking index in a general case of a time-dependent magnetic field can be simply expressed as follows

$n=3-4 \frac{\dot{B_{0}}}{B_{0}} \tau_{\mathrm{c}} \equiv 3-4 \frac{\tau_{\mathrm{c}}}{\tau_{B}}$,

where $\tau_{\mathrm{c}}=P / 2 \dot{P}$ is the characteristic age, and we defined the magnetic field evolution timescale $\tau_{B} \equiv B_{0} / \dot{B}_{0}$. This last equation shows that any variation of the dipolar surface magnetic field strength results in a deviation from the $n=3$ standard value, which is obviously recovered for a constant magnetic field $\left(\dot{B}_{0}=0\right)$. For an increasing $B_{0}$ we will always obtain $n<3$, while $n>3$ is the signature of a decreasing $B_{0}$.

\section{Magnetic field evolution scenarios}

The precise calculation of the time variation of $B_{0}$ requires solving the problem of the coupled magneto-thermal evolution of a neutron star (Pons et al. 2009; Viganò et al. 2012). However, before showing results from simulations, one can qualitatively analyze of the most important physical process at different ages. We now discuss three possible scenarios.

\subsection{Amplification or reemergence of the dipolar surface magnetic field}

The generic $n<3$ observed without exception for young pulsars can be caused by the rediffusion of the magnetic field submerged in the crust during the supernova fallback episode (Young \& Chanmugam 1995; Muslimov \& Page 1995; Geppert et al. 1999). The submergence of the magnetic field has also been studied in recent MHD simulations, with interesting implications for gravitational wave emission (Vigelius \& Melatos 2009; Wette et al. 2010). The rediffusion timescale depends essentially on the total amount of accreted matter. For total accreted masses between $10^{-4}-10^{-3} M_{\odot}$, screening currents are dissipated on $\tau_{\text {rediff }} \sim 10^{3}-10^{4} \mathrm{yr}$, as recent calculations have confirmed (Ho 2011; Viganò \& Pons 2012). The same idea has been applied to the reemergence of magnetic fields in accreting binary systems with similar results (Cumming et al. 2001), and evidence for fast magnetic field evolution in an accreting millisecond pulsar has been reported (Patruno 2012).

Alternative mechanisms are time variations in the angle between the rotational and magnetic axes (Link \& Epstein 1997; Ruderman et al. 1998), or the thermoelectric field generation 
that may proceed in the crust and envelope of young pulsars if a sufficiently strong temperature gradient is present (Urpin et al. 1986; Wiebicke \& Geppert 1996). This process is limited by the condition that the surface temperature of the neutron star should not be lower than $3 \times 10^{6} \mathrm{~K}$ (Wiebicke \& Geppert 1996), which corresponds to about $1000 \mathrm{yr}$ in the standard cooling scenario. In our simulations we included the reemergence of a screened magnetic field, but our present version of the code does not include magnetic field generation by thermoelectric effect, or other possible mechanisms such as magnetic flux expulsion from the superconducting core.

\subsection{Ohmic decay}

Ohmic dissipation of the magnetic field has been thoroughly investigated in the past by many groups (Haensel et al. 1990; Page et al. 2000; Tauris \& Konar 2001). The usual definition of the Ohmic decay time is

$\tau_{\mathrm{Ohm}}=\frac{4 \pi \sigma L^{2}}{c^{2}}$,

where $L$ denotes the scale-length of magnetic field variations and $\sigma$ the electric conductivity. In a neutron star crust, $\sigma$ is dominated by electronic transport and depends on the electron density, the crustal temperature, and the impurity concentration within the crust. Since the electron density varies over about four orders of magnitude in the crust and the temperature decreases by about two to three orders of magnitude in a pulsar's lifetime, the electric conductivity may vary both in space and time by many orders of magnitude (see Fig. 1 in Pons \& Geppert 2007). Therefore, to assume a uniform Ohmic decay time $\tau_{\mathrm{Ohm}}$, independent of the location of currents and the pulsar age is, generally speaking, quite misleading: Ohmic decay in a neutron star crust cannot be described by a single exponential law (see e.g. Page et al. 2000, for a qualitative discussion). Since the conductivity increases with decreasing temperature, Ohmic diffusion becomes an increasingly slow process in older pulsars, beyond the transition from neutrino dominated to photon cooling at $\gtrsim 10^{5} \mathrm{yr}$. The only general fact that we can expect is that, for lowfield middle-aged neutron stars $\left(10^{4}-10^{5} \mathrm{yr}\right)$, this is expected to be the dominant process, thus resulting in $n>3$.

\subsection{Hall drift oscillatory modes}

The Hall drift, through its nonlinear dependence on the magnetic field, has an influence on the magnetic field evolution either for magnetar conditions $\left(B>10^{14} \mathrm{G}\right)$, or alternatively when the electrical resistivity becomes very low. Under certain circumstances the Hall drift can drain magnetic energy out of the dipolar mode and redistribute it into smaller scale ones. This sometimes causes an apparently rapid decrease of $B_{0}$. But the opposite effect may also occur, i.e., pumping energy from an internal strong toroidal field to the dipolar poloidal component. Both scenarios would happen on the Hall timescale

$\tau_{\text {Hall }}=\frac{4 \pi n_{\mathrm{e}} e L^{2}}{c B}$,

where $n_{\mathrm{e}}$ is the electron density and $e$ the elementary charge. For typical values in NSs we have $\tau_{\text {Hall }} \sim\left(10^{4}-10^{6}\right) \frac{10^{14} \mathrm{G}}{B_{0}} \mathrm{yr}$.

In situations of quasi-equilibrium, oscillatory modes with magnetic energy exchange between the different crustal field
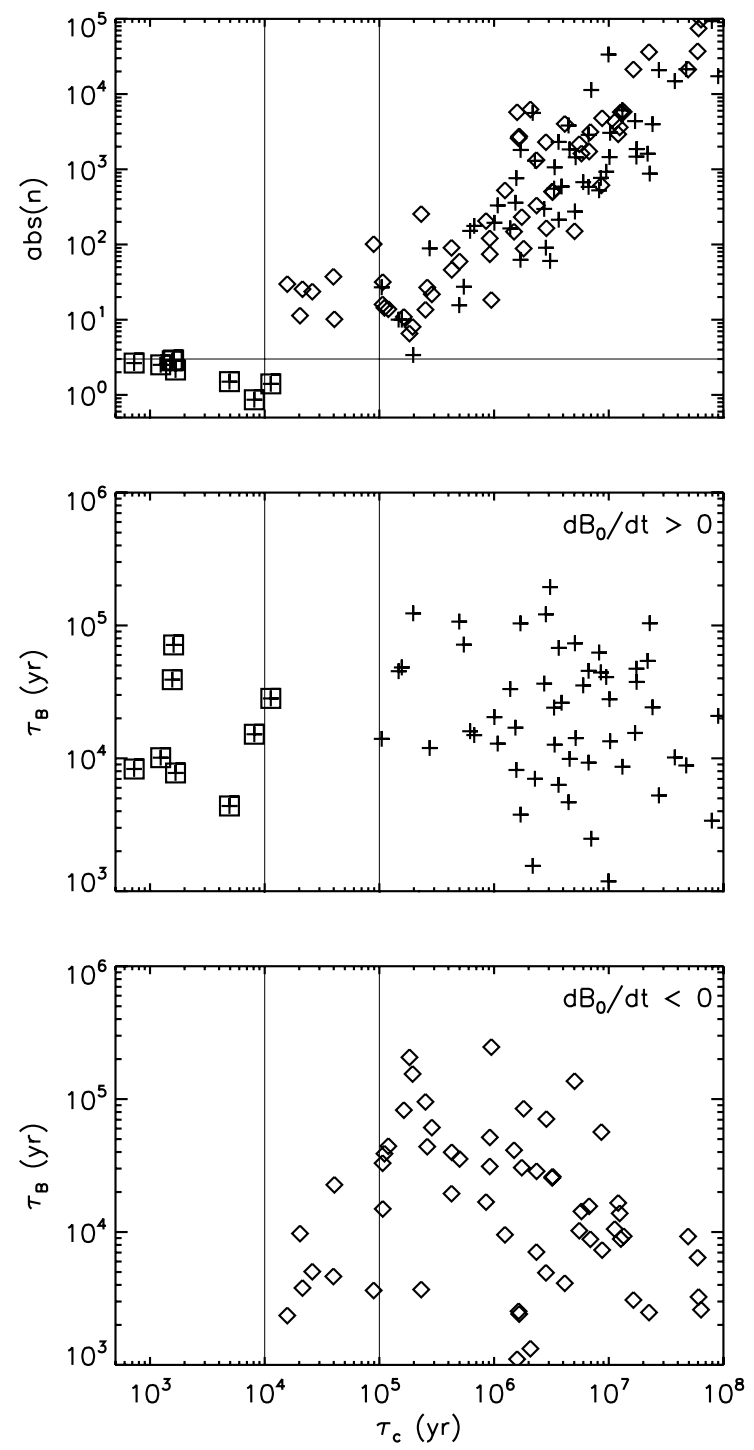

Fig. 1. Top: braking indices (absolute value) as a function of $\tau_{\mathrm{c}}$ for our sample of 118 pulsars. We represent pulsars with $n<3$ with crosses and pulsars with $n>3$ with diamonds. Note that all objects marked with crosses and $\tau_{\mathrm{c}}>10^{5}$ yr have actually $n<0$. The 8 youngest objects discussed in Espinoza et al. (2011) are marked with squares. Middle: magnetic field evolution timescale, $\tau_{B}=4 \tau_{\mathrm{c}} /(3-n)$ for pulsars with $n<3$. Bottom: absolute value of $\tau_{B}$ (they are all negative) for pulsars with $n>3$.

modes are expected. The temporal evolution of the polar surface magnetic field can then be approximated by

$B_{0}(t)=B_{0}+\delta B \sin \left(\frac{2 \pi t}{\tau_{\text {Hall }}}\right)$

and from Eq. (8) we have

$n=3-4 \frac{\dot{B_{0}}}{B_{0}} \tau_{\mathrm{c}} \approx 3-8 \pi \frac{\tau_{\mathrm{c}}}{\tau_{\text {Hall }}} \frac{\delta B}{B_{0}} \cos \left(\frac{2 \pi t}{\tau_{\text {Hall }}}\right)$.

These oscillations are expected in young magnetars with $B_{0}>$ $10^{14} \mathrm{G}$. It causes corrections to the canonical braking index $n=3$ of either positive or negative sign, according to Eq. (12). These corrections are expected to be small because of their short $\tau_{\mathrm{c}}$, and to be increasingly important for objects with smaller $\dot{P}$ (old characteristic ages). In addition, as the star cools down, the drop 
in electrical resistivity may activate the Hall term even for normal pulsars $B_{0} \gtrsim 10^{12}$ and the occurrence of oscillatory modes during a second Hall stage at late times is a natural outcome. First estimates suggest that $\delta B / B_{0}$ could be as large as $\sim 10^{-1}$. When $\tau_{\mathrm{c}} \gg \tau_{\text {Hall }}$, the second term in Eq. (12) dominates and the magnetic field oscillatory modes should result in equally probable positive and negative braking indices with high absolute values. However, this needs to be confirmed by long term realistic simulations.

\section{Discussion}

\subsection{Magnetic field evolution in the pulsar population?}

From the whole population in the ATNF Pulsar Catalogue (Manchester et al. 2005), we extracted a sample of pulsars for which $\ddot{v}$ has a quoted error smaller than $10 \%$. From this preselected sample, we excluded all pulsars in binaries and those with very short periods $(P<15 \mathrm{~ms})$, likely to be recycled, and we doubled checked our list with the more recent review by Hobbs et al. (2010), the first large-scale analysis of pulsar timing noise over timescales $>10 \mathrm{yr}$, which led us to remove several more pulsars whose revised values were inconsistent with the ATNF data, or with larger errors. After this selection, our sample contains 118 radio-pulsars, about half of which have negative braking indices.

We show in the top panel of Fig. 1 the observed distribution of braking indices as a function of $\tau_{\mathrm{c}}$. We considered the characteristic magnetic field evolution timescale, $\tau_{B}=4 \tau_{\mathrm{c}} /(3-n)$ (see Eq. (8)) and separated the sample into two groups, those with positive (middle panel) and negative (bottom panel) $\tau_{B}$. The eight pulsars discussed in Espinoza et al. (2011) are marked with squares. With all due cautions regarding the uncertainties associated to these measurements, it is worth mentioning some interesting trends visible in this plot:

- All young objects seem to have always $n<3$, which can be a hint of an increasing dipolar magnetic field ${ }^{1}$.

- All middle age objects $\left(10^{4}-10^{5} \mathrm{yr}\right)$, except Vela, that survived to our conservative selection criteria have negative time derivatives of $B_{0}^{2}$.

- For old objects, there is no correlation at all, and there are similar numbers of objects with positive and negative derivatives of the field. The typical evolution timescales are in the range $10^{3}-10^{5} \mathrm{yr}$.

The strong correlation between $|n|$ and $\tau_{\mathrm{c}}$ seen in the old objects of the top panel simply reflectis the definition of $n$, Eq. (8), with the additional piece of information that $\tau_{B}$ does not seem to be correlated with $\tau_{\mathrm{c}}$ (see middle and bottom panels).

Note that our criteria automatically select the objects with a dominant contribution of $\ddot{v}$ in the timing phase residuals (i.e., the cleanest cubic lines in Fig. 3 of Hobbs et al. (2010), e.g. B0114+58). This introduces a bias toward objects with high braking indices, and against pulsars with residuals dominated by higher order terms (e.g. B0136+57) or quasi-periodic terms (e.g. B1642-03 or B1826-17) in the time-dependent phase (Lyne et al. 2010). In the latter cases, the estimates of $\ddot{v}$ are subject to

\footnotetext{
1 We use the values collected in Espinoza et al. (2011) for this plot.

2 We did not include seven more objects, that fall in this region, with a quoted error of $\ddot{v}$ smaller than $10 \%$ in the ATNF pulsar database. They are not considered in Hobbs et al. (2010), probably because they did not have $10 \mathrm{yr}$ of accumulated data, although their values have not been reported to change.
}

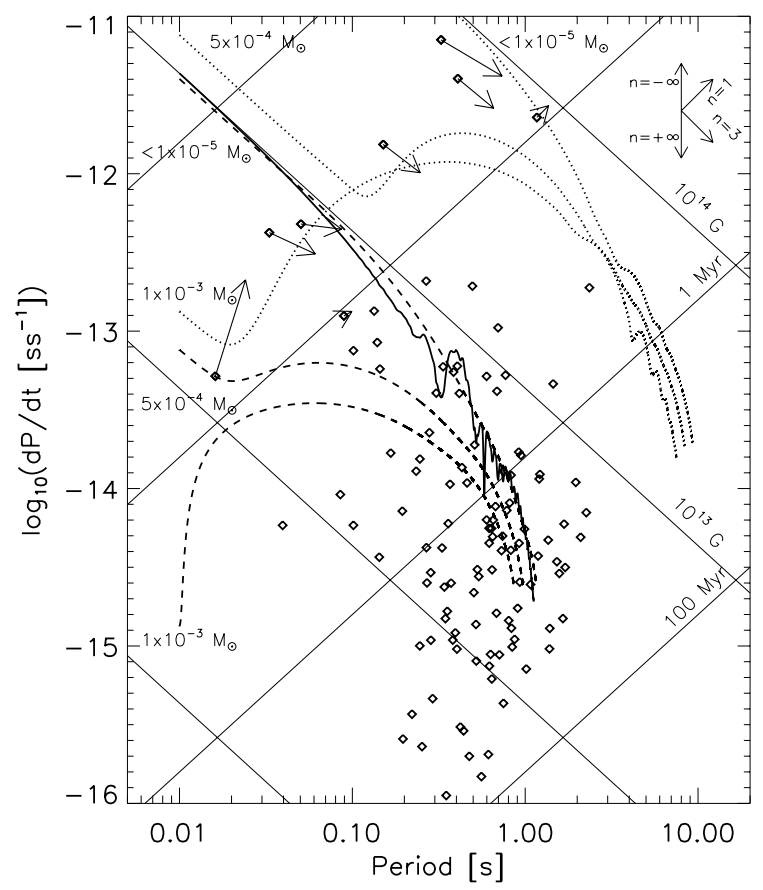

Fig. 2. Evolutionary tracks in the $P-\dot{P}$ diagram during the first $3 \mathrm{Myr}$ for: model A (dashed) and model B (dotted), both for different values of accreted mass $M_{a}=[0.1,5,10] \times 10^{-4} M_{\odot}$; model C (solid) without accretion.

larger uncertainties, and $n$ strongly depends on the analyzed time interval (Hobbs et al. 2010). However, we have checked that the general trends do not change if the sample is enlarged by including pulsars with quoted errors in $\ddot{v}$ up to $80 \%$. This simply increases the statistics ( $>300$ sources) and includes some objects with lower value of $|n-3|$ (shorter $\left.\left|\tau_{B}\right|\right)$.

\subsection{Expected evolution from theoretical models}

We performed a series of numerical simulations with the magneto-thermal evolution code presented in Aguilera et al. (2008); Pons et al. (2009), and Viganò et al. (2012), which includes all relevant microphysical processes and follows the complex feedback between the physical mechanisms presented in a simplified way in the previous section. In addition to Ohmic dissipation and Hall drift effects, which are consistently included in the simulations, we assumed that all models suffer an episode of hypercritical accretion during their formation.

We plot in Fig. 2 the evolutionary tracks of some theoretical models in the $P-\dot{P}$ diagram, compared to the pulsars of our sample. We show results for three initial magnetic field configurations. Two of them are purely dipolar, although during the evolution other multipoles and toroidal field are naturally created. They differ by the magnetic field strengths: $B_{0}=10^{13} \mathrm{G}$ (model $\mathrm{A}$, dashed lines) and $10^{14} \mathrm{G}$ (model $\mathrm{B}$, dotted lines). In each case (models $\mathrm{A}$ and $\mathrm{B}$ ) we compare results with three values of the total accreted mass. The third initial configuration (model C, solid line) has an initial dipolar field of $B_{0}=10^{13} \mathrm{G}$, as model $\mathrm{A}$, but with an additional octupolar component of strength $3.5 \times 10^{14} \mathrm{G}$ (at the pole). This model serves to make explicit the effect of the complex initial geometries. Assuming vacuum external boundary conditions, the toroidal field has to vanish at the star surface, but the dipolar component is still coupled to higher order multipoles, which can also interchange energy with the dipolar mode. 
For the eight youngest pulsars, we indicate their predicted movement for the next $2.5 \mathrm{kyr}$ with arrows, assuming the present value of $n$, from Espinoza et al. (2011), remains constant. The direction of the tangent vector to a given track is related to the braking index. For reference, we indicate in the legend on the upper right corner that $n=3$ and $n=1$ imply constant inferred $B_{0}$, and constant characteristic age, respectively.

The initial period $P_{\text {in }}$, which was assumed to be $0.01 \mathrm{~s}$ in all cases, only affects the early stage, while $\dot{P} \lesssim P_{\text {in }} / t$ (with $t$ being the real age), and $P \simeq P_{\text {in }}$. Models with deep submergence of the field but with different initial periods also have vertical trajectories shifted to the left/right depending on $P_{\text {in }}$, and quickly cross the range of $\dot{P}$ where the bulk of pulsars lie. At late times, tracks coming from the same model but with different $P_{\text {in }}$ are indistinguishable, typically converging after $t \sim 30\left(\frac{P_{\text {in }}}{0.01 s} \frac{10^{13} \mathrm{G}}{B_{\text {in }}}\right)^{2} \mathrm{yr}$, with $B_{\text {in }}$ being the initial magnetic field. Therefore, $P_{\text {in }}$ has an appreciable long-term effect only in the deep submergence case $\left(M_{a} \gtrsim 10^{-3} M_{\odot}\right)$, for which $B_{\text {in }}$ is strongly reduced: in these models, the first few $10^{4} \mathrm{yr}$ are spent in the vertical trajectories, with $n \ll 0$. Note that in this reemergence phase there is no correlation between the real and characteristic ages.

When reemergence of the field has almost been completed, the trajectories reach the high- $\dot{P}$ region (i.e., largest $B_{0}$ ) and progressively bend. The more extreme braking indices of PSR J1734-3333 $(n=0.9 \pm 0.2$, Espinoza et al. 2011) and PSR J0537-6910 ( $n \sim-1.5$, Middleditch et al. 2006) would be consistent with the last stage of the reemergence after a deep submergence into the inner crust. On the other hand, in the shallow submergence models $\left(M_{a} \sim 10^{-5}-10^{-4} M_{\odot}\right)$, after accretion stage $B_{0} \simeq B_{\text {in }}$. These tracks initially run almost along the isomagnetic lines: pulsars with $n$ slightly less than 3 are compatible with this scenario.

Independently of the early reemergence phase (if any), tracks with the same $B_{\text {in }}$ converge at middle-age, and have slopes corresponding to $n>3$, characteristic of the slow Ohmic dissipation. During this phase, there is a correlation between real and characteristic ages, with typically $\tau_{c}$ being a factor of few longer than the real age. Some tracks show visible oscillations produced by the Hall activity when the star is cold enough $\left(t \gtrsim 10^{5} \mathrm{yr}\right)$; in particular, model $\mathrm{C}$ (solid line) clearly shows that any complex initial geometry may have a distinct signature on the timing properties of pulsars.

We emphasize that we did not attempt to fit individual objects: our purpose with this sample of models is simply to show that, with reasonable assumptions, it is possible to explain the variability in the observed range of braking indices of young pulsars and to predict their evolutionary paths in the $P-\dot{P}$ diagram.

\subsection{Braking index and evolution timescale for realistic magnetic field evolution models}

In Fig. 3 we plot the braking index evolution for three representative models, Note that the horizontal axis in Fig. 1 represents the characteristic age $\tau_{\mathrm{c}}$, while in Fig. 3 we show our results as a function of the real age of each model, so that a direct comparison is not possible. We also used a linear scale in this plot to show the quasi-periodic oscillations during the long-term evolution more clearly. For fields $\lesssim 10^{13} \mathrm{G}$ and simple dipolar geometries (dashed line) the braking index at late times is $n>3$, but its absolute value is low. In contrast, for strong dipolar fields, or for weak dipolar components but with strong higher order multipoles, Hall-drift induced oscillations appear sooner or later and, in some situations, have large amplitudes that result in very high

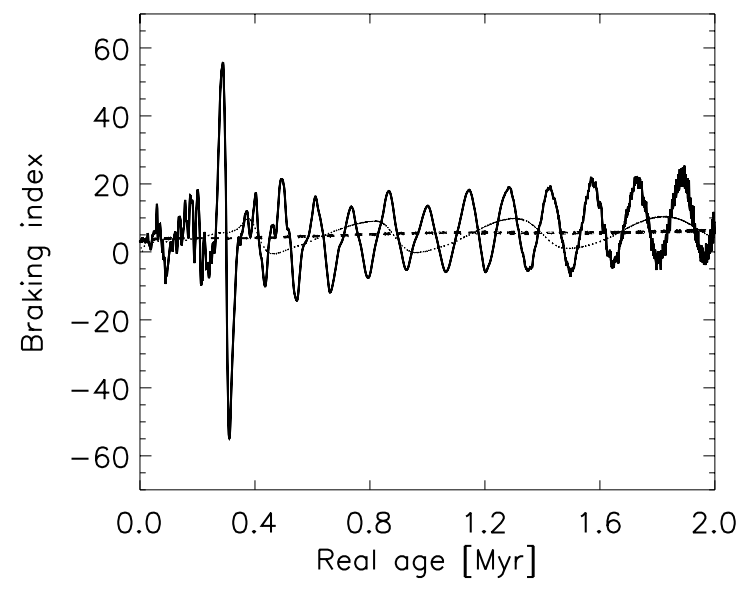

Fig. 3. Braking index $(n)$ as a function of age for model $\mathrm{A}$ (dashed line), model B with $M_{a}=10^{-3} M_{\odot}$ (thin solid line), and model C (thick solid line).

absolute values of the braking index. It is particularly interesting to compare models A and C (dashed and solid line), which have the same initial dipolar component, representative of a typical pulsar (at $10^{6} \mathrm{yr}$ the dipolar field is about $\left.3-5 \times 10^{12} \mathrm{G}\right)$. However, the presence of a strong octupolar component at birth results in a radically different braking index behaviour, even if $P$ and $\dot{P}$ are similar. The amplitude of the oscillations and whether the modes are damped or excited depends on particular details of the smallscale structure of the magnetic field, which is unknown. The exact age at which these oscillatory modes are excited is connected to the temperature of the star, and therefore to its cooling history and internal physics (neutrino emission processes, superfluid gaps, etc.). For the standard cooling scenario this happens at $\approx 10^{5} \mathrm{yr}$. We can also observe that as the star evolves and the magnetic diffusivity decreases, the frequency of the dominant mode may vary (see solid line). For example, at early times in model $\mathrm{C}$ we observe variability on shorter timescales (few kyr), while later we see longer oscillation periods $\left(10^{5} \mathrm{yr}\right)$. After 1.5 Myr higher frequency modes seem to be growing again.

\subsection{Timing residuals}

In timing analysis of radio pulsars, an important piece of information is the study of the residuals, i.e., the phase differences between the observed signal and the best-fit model, including the frequency and frequency derivative. As shown in detail in Hobbs et al. (2010), there is a rich variety of shapes in the residuals of radiopulsars. As we have shown, the strong Hallinduced interplay between different multipoles and the toroidal field produces a complex evolution of $B_{0}$. To compare with observational timing analysis, we proceeded as follows: first, we choose a short interval of $40 \mathrm{yr}$ in our simulations, centered on a fixed time denoted by $t_{0}$. This interval is similar to the longest periods for which phase-coherent timing analysis for radio pulsars can be performed. Then, from our theoretical $B_{0}(t)$, we obtain $v(t)$ by integration of the classical spindown formula (Eq. (6)) assuming an orthogonal rotator, and the phase $\Phi(t)=\int_{t_{0}}^{t} v\left(t^{\prime}\right) \mathrm{d} t^{\prime}$. Finally, we fit our synthetic time-dependent phase with a quadratic function

$\Phi(t)=\Phi_{0}+v_{f}\left(t-t_{0}\right)+\frac{1}{2} \dot{v}_{f}\left(t-t_{0}\right)^{2}$.

The results of the fit, $v_{f}$ and $\dot{v}_{f}$, are of course in the range of values of the real quantities in the time interval. 

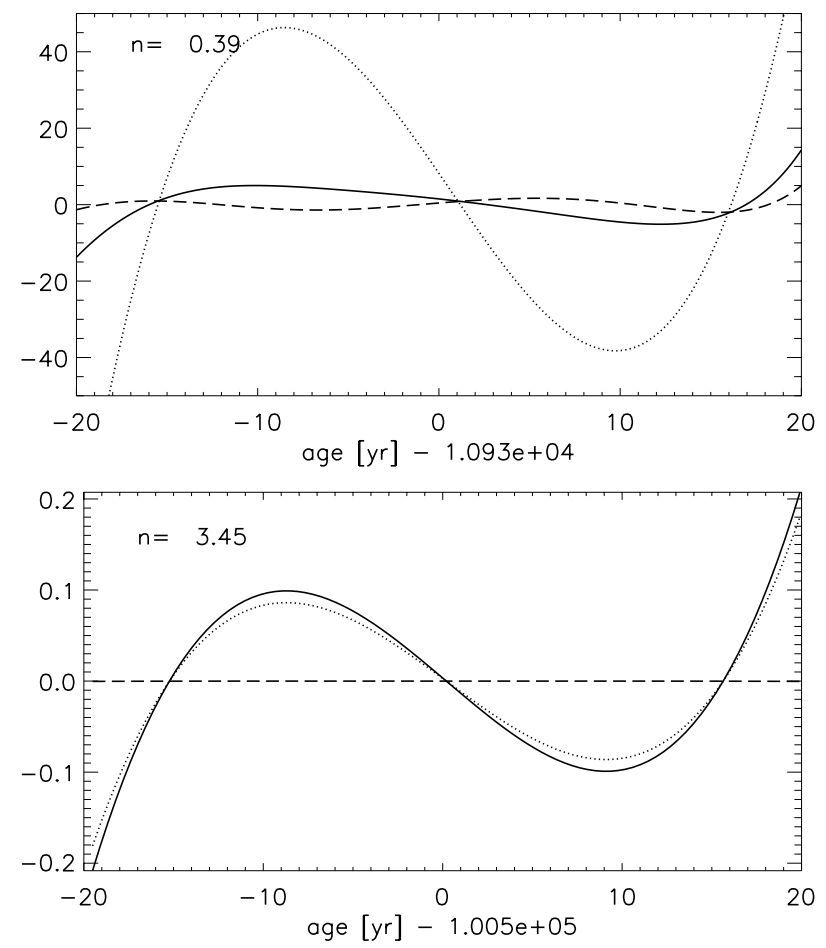

Fig. 4. Phase residuals for model C around $t_{0}=10.93 \mathrm{kyr}$ (upper panel) and $t_{0}=100.5 \mathrm{kyr}$ (lower panel), with the corresponding value of $n$ indicated in each case. We show residuals after removing the best-fit solution including quadratic terms (solid) or including cubic terms (long dashes); dotted lines show the cubic residuals obtained assuming a constant $B_{0}$.

In Fig. 4 we show the phase residuals for model $\mathrm{C}$ (solid lines) at $t_{0}=10.93 \mathrm{kyr}$ (top) and $t_{0}=100.5 \mathrm{kyr}$ (bottom). During the two analyzed intervals, the mean values of the dipolar magnetic field are $\bar{B}_{0}=8.1 \times 10^{12} \mathrm{G}$ and $\bar{B}_{0}=5.3 \times 10^{12} \mathrm{G}$. The cubic shape indicates that the residuals are dominated by the next term in the Taylor series (i.e., red noise). Including in the fitting function a cubic term allows one to measure $\ddot{v}$ and the braking index ( $n=0.39$ and $n=3.45$, respectively). With dashes we show the fourth-order residuals after subtracting the third-order term in the fitting function. For comparison, we also show with dotted lines the third-order residuals obtained assuming a constant value $B_{0}(t)=\bar{B}_{0}$ (that leads to $n=3$ ).

In the first time interval, the change of magnetic field, $\delta B_{0}=$ $1.3 \times 10^{10} \mathrm{G}=1.6 \times 10^{-3} \bar{B}_{0}$, is strong enough for the cubic residuals to be visibly different from the constant field case. The value of $|\ddot{v}|$ (and of $|n|$ ) is low, and the residuals of fourth order are significant. In the second time interval, $\delta B_{0}=1.4 \times 10^{8} \mathrm{G}=$ $2.6 \times 10^{-5} \bar{B}_{0}$, and $n$ is close to 3 . As a consequence, the deviation from the constant field spin-down behavior is slow and fourthorder residuals are orders of magnitude weaker (dashed line).

To investigate the effect of short-time irregularities, we repeated the process but artificially added two additional sources of "noise" $\left(B_{p}\right)$ to our theoretical values of $B_{0}(t)$, with amplitude of the same order as the theoretical variations $\delta B_{0}$. In Fig. 5 we show the residuals obtained by adding a sinusoidal perturbation, $B_{p}=\left(\delta B_{0} / 2\right) \sin (2 \pi t / T)$, with $T=10 \mathrm{yr}$ (dashed lines), or a random perturbation of maximum absolute value $\delta B_{0} / 2$ (dashdotted lines). In our analysis, we sampled values every $\sim 10^{-2} \mathrm{yr}$, comparable with the typical integration time in observational data. We checked that increasing the time interval strongly reduces the contribution of the random noise at a fixed amplitude, as expected. Similarly, the periodic short-term noise is reduced

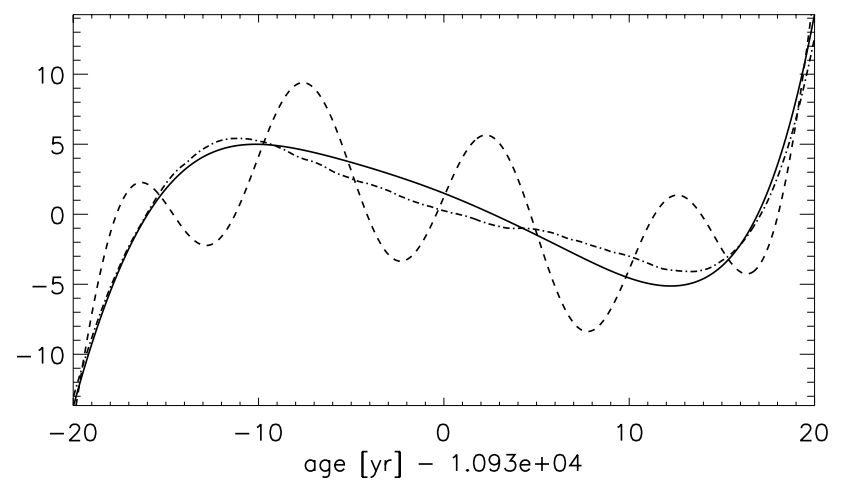

Fig. 5. Comparison of the cubic residuals of the theoretical model (solid line) with the result after adding a sinusoidal perturbation with frequency $0.1 \mathrm{yr}^{-1}$ (dashed) or a random perturbation (dot-dashed). For both perturbation forms we considered a maximum amplitude of $\left(\delta B_{0} / 2\right)=6.5 \times 10^{9} \mathrm{G}$.

when the integration interval becomes much longer than the perturbation period.

Note that small perturbations of $B_{0}$ produce visible effects, but the cubic residual still dominates. In constrast, in many pulsars a smooth cubic phase residual is clearly seen, which means that the spin-down is very stable, short-term irregularities are completely negligible (or averaged out in the long observation period), and the measure of a braking index (even a high value) dominated by the secular evolution is robust. Glitches should appear as cusps in these plots (e.g. see data for PSR B0154+61 in Hobbs et al. 2010), but a more detailed investigation in this direction is beyond the scope of this paper.

\section{Conclusions}

According to our current understanding of the magneto-thermal evolution of neutron stars, the time variation of the magnetic field notably affects the braking index of pulsars and contributes to the so-called red noise in the timing residuals. The qualitative picture seems to agree with the data: i) there is a short stage in which the dipolar field appears to be increasing, possibly reflecting the reemergence of magnetic field after initial accretion; ii) a stage dominated by Ohmic dissipation, while the star is still warm, and during which we always expect $n>3$; iii) once the star has cooled down, after about $10^{5} \mathrm{yr}$, oscillatory Hall-drift modes in the crust are excited, and quasi-periodic oscillations of the braking index are consequently expected.

This is by no means the only contribution to the timing noise, nor necessarily the dominant one. For example, changes in the effective moment of inertia as the superconducting region of the core grows (Ho \& Andersson 2012) and unknown mechanisms for alignment of the rotation and magnetic field (Tauris \& Manchester 1998; Young et al. 2010) would have similar longterm effects. In addition to this secular evolution, short-term magnetospheric effects, such as oscillations of the inclination angle around the equilibrium position, or magnetospheric changes (Li et al. 2012a,b), could be also responsible for the strong quasiperiodic features seen in phase residuals for several cases (Lyne et al. 2010).

Our results show that realistic magnetic field evolution models predict temporal variations consistent with the observed trends. In particular, configurations similar to our model $\mathrm{C}$ could explain the wide range in positive and negative values that are observed. This model has a moderate dipolar component $\left(10^{13} \mathrm{G}\right.$ at birth, which becomes a few times $10^{12} \mathrm{G}$ at middle age), typical of pulsars, but a strong octupolar component, large enough 
to activate Hall modes. The same generic behavior is expected for other models with strong, smaller scale components, even if the dipole is weak. However, it must be mentioned that the models we studied predict high values of the braking index (10-100) but not extremely high values $\left(10^{3}-10^{4}\right)$, as reported for pulsars with old characteristic ages. The absolute value of the braking index at late times, which can be of either sign, is not simple to predict, since it depends on particular details of important components of the magnetic field (higher order multipoles, toroidal component). Perhaps different field geometries (e.g., without axial symmetry) and/or magnetospheric corrections may account for the discrepancy.

We emphasize that there is absolutely no reason to expect that the magnetic field remains constant during a pulsar lifetime, and we must abandon the oversimplified models that assume constant (in time) pure dipolar fields. On the other hand, since there is a strong interplay between the magnetic, thermal, and rotational evolution of neutron stars, there are unexpected ways to obtain information. If future observations (or a deeper analysis of archival data) increase the number of reliable measures of braking indices, and we can firmly establish at which age the transition from the purely diffusive to the oscillatory regime happens, there is coded information about the cooling history of neutron stars of potential interest to place constraints on the interior physics. In this respect, it is also important to mention that precise phase-connected timing solutions can also be performed in other bands, as has been recently done for $17 \gamma$-ray-selected pulsars (Ray et al. 2011), using the Large Area Telescope (LAT) on the Fermi Gamma-ray Space Telescope. This and other future high-energy missions can enlarge our data sample and improve the precision of our measurements.

Acknowledgements. This research was supported by the grants AYA 201021097-C03-02 and ACOMP/2012/135. D.V. is supported by a fellowship from the Prometeo program for research groups of excellence of the Generalitat Valenciana (Prometeo/2009/103). We thank C. Espinoza and J. A. Miralles for their valuable comments.

Note added in proof. After the acceptance of this article for publication, we became aware of two new papers closely related to our results by Shuang-Nan Zhang \& Yi Xie (2012a,b), with similar conclusions.

\section{References}

Aguilera, D. N., Pons, J. A., \& Miralles, J. A. 2008, A\&A, 486, 255 Barsukov, D. P., \& Tsygan, A. I. 2010, MNRAS, 409, 1077
Beskin, V. S., \& Nokhrina, E. E. 2007, Ap\&SS, 308, 569

Cumming, A., Zweibel, E., \& Bildsten, L. 2001, ApJ, 557, 958

Espinoza, C. M., Lyne, A. G., Kramer, M., Manchester, R. N., \& Kaspi, V. M. 2011, ApJ, 741, L13

Geppert, U., Page, D., \& Zannias, T. 1999, A\&A, 345, 847

Haensel, P., Urpin, V. A., \& Iakovlev, D. G. 1990, A\&A, 229, 133

Haskell, B., Jones, D. I., \& Andersson, N. 2006, MNRAS, 373, 1423

Ho, W. C. G. 2011, MNRAS, 414, 2567

Ho, W. C. G., \& Andersson, N. 2012 [arXiv: 1208. 3201]

Hobbs, G., Lyne, A. G., \& Kramer, M. 2010, MNRAS, 402, 1027

Li, J., Spitkovsky, A., \& Tchekhovskoy, A. 2012a, ApJ, 746, L24

Li, J., Spitkovsky, A., \& Tchekhovskoy, A. 2012b, ApJ, 746, 60

Link, B., \& Epstein, R. I. 1997, ApJ, 478, L91

Livingstone, M. A., Kaspi, V. M., Gavriil, F. P., et al. 2007, Ap\&SS, 308, 317

Livingstone, M. A., Ng, C.-Y., Kaspi, V. M., Gavriil, F. P., \& Gotthelf, E. V. 2011, ApJ, 730, 66

Lyne, A. G. 2009, in Astrophysics and Space Science Library, ed. W. Becker, 357,67

Lyne, A. G., Pritchard, R. S., \& Graham-Smith, F. 1993, MNRAS, 265, 1003

Lyne, A. G., Pritchard, R. S., Graham-Smith, F., \& Camilo, F. 1996, Nature, 381, 497

Lyne, A., Hobbs, G., Kramer, M., Stairs, I., \& Stappers, B. 2010, Science, 329, 408

Manchester, R. N., Hobbs, G. B., Teoh, A., \& Hobbs, M. 2005, AJ, 129, 1993

Middleditch, J., Marshall, F. E., Wang, Q. D., Gotthelf, E. V., \& Zhang, W. 2006, ApJ, 652, 1531

Muslimov, A., \& Page, D. 1995, ApJ, 440, L77

Page, D., Geppert, U., \& Zannias, T. 2000, A\&A, 360, 1052

Patruno, A. 2012, ApJ, 753, L12

Pons, J. A., \& Geppert, U. 2007, A\&A, 470, 303

Pons, J. A., Miralles, J. A., \& Geppert, U. 2009, A\&A, 496, 207

Ray, P. S., Kerr, M., Parent, D., et al. 2011, ApJS, 194, 17

Ruderman, M., Zhu, T., \& Chen, K. 1998, ApJ, 492, 267

Shibazaki, N., \& Mochizuki, Y. 1995, ApJ, 438, 288

Spitkovsky, A. 2006, ApJ, 648, L51

Tauris, T. M., \& Konar, S. 2001, A\&A, 376, 543

Tauris, T. M., \& Manchester, R. N. 1998, MNRAS, 298, 625

Urama, J. O., Link, B., \& Weisberg, J. M. 2006, MNRAS, 370, L76

Urpin, V. A., Levshakov, S. A., \& Iakovlev, D. G. 1986, MNRAS, 219, 703

Viganò, D., \& Pons, J. A. 2012, MNRAS, 425, 2487

Viganò, D., Pons, J. A., \& Miralles, J. A. 2012, Comput. Phys. Comm., 183, 2042

Vigelius, M., \& Melatos, A. 2009, MNRAS, 395, 1985

Weltevrede, P., Johnston, S., \& Espinoza, C. M. 2011, MNRAS, 411, 1917

Wette, K., Vigelius, M., \& Melatos, A. 2010, MNRAS, 402, 1099

Wiebicke, H., \& Geppert, U. 1996, A\&A, 309, 203

Young, E. J., \& Chanmugam, G. 1995, ApJ, 442, L53

Young, M. D. T., Chan, L. S., Burman, R. R., \& Blair, D. G. 2010, MNRAS, 402,1317

Zhang, S.-N., \& Xie, Y. 2012a, ApJ, 757, 153

Zhang, S.-N., \& Xie, Y. 2012b, ApJ, submitted [arXiv: 1209.2478] 